\title{
Review of: "Prevention of suicidal behavior in older people: A systematic review of reviews"
}

madia lozupone, Francesco Panza

Potential competing interests: The author(s) declared that no potential competing interests exist.

Commentary on: Prevention of suicidal behavior in older people:

A systematic review of reviews

Madia Lozupone ${ }^{1}$ and Francesco Panza ${ }^{2}$

${ }^{1}$ Neurodegenerative Disease Unit, Department of Basic Medicine, Neuroscience, and Sense Organs, University of Bari Aldo Moro, Bari, Italy

2 Unit of Research Methodology and Data Sciences for Population Health, National Institute of Gastroenterology "Saverio de Bellis", Research Hospital, Castellana Grotte, Bari, Italy

Text word count: 912

References: 9

\section{Correspondence:}

Madia Lozupone, MD, PhD

Neurodegenerative Disease Unit,

Department of Basic Medicine,

Neuroscience, and Sense Organs,

University of Bari Aldo Moro, Bari, Italy

Email: madia.lozupone@gmail.com

ORCID iD: 0000-0002-1674-9724

Most suicide attempters give warning signs to family members, not to professionals. Signs are often missed 
among older adults, for whom talking about death and dying is often considered normal. In older age, efforts for suicide prevention should be based on strategies to assess and treat risk factors. However, a clear recognition on the risk factors for suicide attempts and completed suicides in older age has not been done yet. A risk factor highly associated with completed suicide in older individuals has been affective illness and, in particular, depression. Mood disorder was significantly more common in older victims. Given the pivotal role of depression as a risk factor for suicide, improvements must be made in the detection and proper treatment of this disorder. In addition, older Australian men with multimorbidity had the highest risk of death by suicide, even after taking into account the presence of mood disorders [1]. The role of depression in late-life suicide deserves particular interest due to its elevated prevalence and relationship with functional disability and chronic disease development [2].

Recently, a systematic review of reviews was conducted about interventions aimed at preventing and/or reduce suicidal behavior (suicide, suicide attempt, self-harm and suicidal ideation) in older people by Laflamme and colleagues [3]. A systematic database search was conducted in eight electronic databases from inception to April 2020 for reviews targeting interventions among adults of 60 years and plus both pharmacological and not-pharmacological. Four high quality reviews were included and interventions categorized as pharmacological (antidepressant use: 239 randomized controlled trial -RCTs-, seven observational studies) and behavioral (physical activity: three observational studies, and multifaceted primary-care-based collaborative care for depression screening and management, four RCTs). The results of this review of reviews find the evidence inconclusive towards use of antidepressants for the prevention of suicidal behavior in older people, thus monitoring is required prior to start, dosage variation or stop of antidepressants. The Authors concluded that evidence to date supports physical activity and collaborative management for reduction of suicide ideation, but additional trials are required for a meta-analysis.

Contrasting evidence came from population-based setting about risk factors for suicide attempts/completed suicides in older age. Where should the focus of intervention be sought? First major concern about the present systematic review is the different target populations among two foci of intervention (pharmacological and not). While pharmacological treatment about antidepressant utilization was made on people affected by depressive disorders, behavioral interventions are evaluated in mental health subjects free of psychiatric disorders. In a recent systematic review, from 39 population-based studies selected in six different databases until February 2021, the most frequent 28 risk factors for suicidal behavior were analyzed. The risk factors more associated to suicide attempts than other variables frequently related to suicidal behavior in older age were: depressive disorders, methods employed to selfharm (particularly poisoning), and psychotropic drug utilization followed by psychological factors and disability. Moreover, male sex, violent methods to self-harm, any psychiatric disorder (depression, anxiety and bipolar disorders), a poor medical condition, stressors/bereavement, and living alone appeared to be more significant for predicting completed suicides in late life [4].

The FDA carried out extensive meta-analyses of randomized, placebo-controlled trials of modern antidepressants in juveniles and both young and older adults (see http://www.fda.gov/cder/ 
drug/antidepressants/default.htm), and a similar analyses of large clinical cohort studies of patients treated with antidepressants or not was reported by Barbui and his colleagues [5]. Both analyses suggest a previously overlooked age-effect, in that antidepressant treatment may somewhat increase risk of suicidal thoughts and perhaps behaviors in juveniles, but may exert substantial antisuicidal effects in older adults, even though overall suicidal risks with antidepressants and placebos were very similar, without stratification by age [6]. About the relationship between mental health and suicidal behaviour, it is possible that a large proportion of the subjects not in contact with mental health services display significant psychiatric symptoms. Unrecognized depression is common in older age, and the present analysis likely underestimated the true magnitude of the association between depression and suicide itself [7]. According to the precision medicine initiative, researchers/clinicians of geriatric sub- specialties (i.e., old age psychiatrists, old age nutritionists, gerodontologists, etc), after a screening with general suicide index scores models, may need to use specific frailty phenotypes to more accurately predict effective treatments and prevention strategies. Psychiatrists frequently assess suicidal patients who present life-threatening emergencies. Psychiatrists, unlike other physicians, do not have laboratory tests and sophisticated diagnostic instruments to assess patients at risk for suicide. For example, when evaluating an emergency cardiac patient, the clinician can order a number of diagnostic tests and procedures, e.g., electrocardiogram, serial enzymes, imaging catherization. The psychiatrist 's diagnostic instrument is competent suicide risk assessment.

Data helping to determine the mechanisms underlying the consequences of environmental and epigenetic roles on brain plasticity and mental health in humans are lacking, particularly in older age groups. But some interventions on epigenetic mechanisms are possible as a means of preventing rather than predicting suicidal behavior in older age. Among these, a healthy diet and lifestyle are associated with slower extrinsic epigenetic age acceleration in blood [8,9].

It is also important to focus on helping older people to cope with psychosocial stress linked to chronic disabilities [10], even apart from psychiatric illness. In the next future, the present findings could help old age psychiatrists to target the prevention of suicidal behavior in late life in people with serious mental disorders and significant age-related multimorbidity and frailty that often complicates the management of mental health in older age.

\section{Conflicts of interest}

Authors have no competing interests to disclose for the present commentary. 


\section{Authors' contributions}

Madia Lozupone and Francesco Panza: conceptualization and writing of the manuscript.

\section{References}

1. Almeida OP, McCaul K, Hankey GJ, Yeap BB, Golledge J, Flicker L, 2016. Suicide in older men: the health in men cohort study (HIMS). Prev. Med. 93, 33-38. https://doi.org/10.1016/j.ypmed.2016.09.022.

2. Fernandez-Rodrigues V, Sanchez-Carro Y, Lagunas LN, Rico-Uribe LA, Pemau A, Diaz-Carracedo P, DiazMarsa M, Hervas G, de la Torre-Luque A. Risk factors for suicidal behaviour in late-life depression: A systematic review. World J Psychiatry. 2022 Jan 19;12(1):187-203. doi: 10.5498/wjp.v12.i1.187.

3. Laflamme L, Vaez M, Lundin K, Sengoelge M. Prevention of suicidal behavior in older people: A systematic review of reviews. PLoS One. 2022 Jan 25;17(1):e0262889. doi: 10.1371/journal.pone.0262889.

4. Beghi M, Butera E, Cerri CG, Cornaggia CM, Febbo F, Mollica A, Berardino G, Piscitelli D, Resta E, Logroscino G, Daniele A, Altamura M, Bellomo A, Panza F, Lozupone M. Suicidal behaviour in older age: A systematic review of risk factors associated to suicide attempts and completed suicides. Neurosci Biobehav Rev. 2021 Aug;127:193-211. doi: 10.1016/j.neubiorev.2021.04.011

5. Barbui C, Esposito E, Cipriani A. Selective serotonin reuptake inhibitors and risk of suicide: a systematic review of observational studies. CMAJ 2009; 180: 291-297.

6. Baldessarini RJ, Tondo L, Strombom I, Dominguez S, Fawcett J, Oquendo M, Licinio J, Valuck R, Tollefson G, Tohen M. Analysis of ecological studies of relationships between antidepressant utilization and suicidal risk. Harv Rev Psychiatry 2007: 15: 133-145

7. Conwell Y, Van Orden K, Caine ED, 2011. Suicide in older adults. Psychiatr. Clin. North Am. 34, 451-468. https://doi.org/10.1016/j.psc.2011.02.002

8. Kim Y, Huan T, Joehanes R et al. Higher diet quality relates to decelerated epigenetic aging. Am. J. Clin. Nutr. 16, nqab201 (2021).

9. Lozupone M, Mollica A, Berardino G, Sardone R, Panza F. Could epigenetics play a role in suicidal behavior in older age? Epigenomics. 2022 Jan;14(2):73-79. doi: 10.2217/epi-2021-0390.

10. Lavretsky H, Newhouse PA. Stress, inflammation, and aging. Am J Geriatr Psychiatry. 2012 Sep;20(9):729-33. doi: 10.1097/JGP.0b013e31826573cf 